\title{
Assessment of fetal and maternal outcome between 40 to 42 weeks and beyond 42 weeks of gestation
}

\author{
Khushboo Yasmin ${ }^{1}$, Ranu Singh Kushwaha ${ }^{2 *}, \mathrm{~K} \mathrm{Jha}^{3}$, Asha Jha ${ }^{4}$ \\ ${ }^{1}$ Junior Residents², Junior Residents, ${ }^{3}$ Professor and Unit Head, ${ }^{4}$ Professor and HOD, Department of Gynecology, Darbhanga Medical College \\ and Hospital. Bihar, INDIA. \\ Email: dr.ranu2010@gmail.com
}

Abstract Background: This study was to assess the fetal and maternal outcome between 40 to 42 weeks and beyond 42 weeks. Prolongation of pregnancy has been the cause of concern for the clinicians and the parturients alike. The pregnancies continuing beyond expected period of confinement. 'postdate', have been found to be associated with various adverse fetomaternal outcomes. Methods: This study was a Prospective, Analytical. Hospital bases study. Was carried out at Darbhanga Medical College and Hospital, Bihar. The study period was twelve months from July 2018 to June 2019. The study population included 402 obstetric cases with Gestational age between 40 to 42 week and beyond 42 weeks who were admitted in Darbhanga Medical College and Hospital, comparison was done for feto-maternal outcomes in between the control group and also between the two groups of postdated pregnancy one is post-dated but term another is post-dated which is truly post-term. Results: Amongst them ninety nine eases (56.6\%) were admitted with gestational age of (40 to 42) weeks and seventy six cases $(4.1 .4 \%)$ weeks period of gestation. Majority of patients in postdate group were nulliparous and more than half of them were between the age group of 19-24years. Most of the patients had spontaneouson set of labour. Induction had to be done in most cases with pregnancy beyond 42 weeks. Conclusion: Adverse maternal like caesarean section, instrumental delivery, postpartum haemorrhage and perineal injury was found to higher in postdate group. These were more seen in mother's beyond 42 weeks period of gestation than 40 weeks to $42^{\text {nd }}$ weeks. However, a larger scale study is recommended to study the accurate pregnancy outcome.

Key Word: Induction of labour, Maternal and fetal complications, Postdate.

*Address for Correspondence:

Dr Ranu Singh Kushwaha, Junior Residents, Department of Gynecology, Darbhanga Medical College and Hospital. Bihar, INDIA.

Email: dr.ranu2010@gmail.com

Received Date: 04/11/2019 Revised Date: 20/12/2019 Accepted Date: 28/01/2020

DOI: https://doi.org/10.26611/10121512

This work is licensed under a Creative Commons Attribution-NonCommercial 4.0 International License. (cc)) EY-NC

\begin{tabular}{|l|l|}
\hline \multicolumn{2}{|c|}{ Access this article online } \\
\hline Quick Response Code: & Website: \\
\hline & www.medpulse.in \\
\hline
\end{tabular}

\section{INTRODUCTION}

Normal duration of pregnancy has been defined by World Health Organization (WHO) and International Federation of Gynecologists and Obstetricians (FIGO) to be lasting between 259 to 294 days of pregnancy from the last menstrual period ${ }^{1}$. Pregnancy that has extended to or beyond 42 weeks of gestation (294 days, or estimated date of delivery [EDD]+14 days) is referred to as post term pregnancy $^{2}$. Other terms that ore used interchangeably arc postdate, prolonged pregnancy or Post-maturity etc. Prolonged pregnancy (i.e. pregnancy beyond expected date of delivery) has always been a matter of anxiety and concern for the pregnant woman and the Obstetricians The delirious effect of pregnancy continuing beyond expected date of delivery was suspected first by Ballantyne in as early as beginning of last century ${ }^{3}$. Recently, studies have shown that obesity and ethnicity also have some role to play in this condition ${ }^{4}$. The incidence of prolonged 
pregnancy varies from $4 \%$ to $14 \%$ in different Studies conducted in various continents. ${ }^{[5]}$ Similar study conducted in Nepal over a period of 3 years from 2005 to 2008 by R Marahatta et al. have found that around $4.6 \%$ of pregnancies reach post term period ${ }^{6}$. Studies conducted in European countries like Sweden ${ }^{7}$ and Russia ${ }^{8}$ were $7.6 \%$ and $3.1 \%$ respectively. Incidence various in these studies possibly due the methods employed to estimate the expected date of delivery (EDO). If early ultrasound scan (USG) along with LMP is used to estimate gestational age then the incidence of 'true' prolonged pregnancy is decreased by a factor of $50 \%$ than when calculated by LMP (last menstrual period) alone. ${ }^{9}$ Moreover, it has been postulated that biological variations like delay in ovulation can result in erroneous over-estimation of gestation age, even though the LMP is known accurately. ${ }^{10}$ Recent, ACOG guidelines however suggests that, if the difference between EDD calculated by these two methods is more than \pm 7 days upto 20 weeks, \pm 14 days upto 20-30weeks and \pm 21 days beyond 30 weeks, then dating by USG should be preferred ${ }^{2}$.

\section{METHODS}

This study was a Prospective, Analytical. Hospital bases study. Was carried out at Darbhanga Medical College and Hospital, Bihar. The study period was twelve months from Nov 2015 to Nov 2016. The study population included 402 obstetric cases with Gestational age between 40 to 42 week and beyond 42 weeks who were admitted in Darbhanga Medical College and Hospital,comparison was done for feto-maternal outcomes in between the control group and also between the two groups of post dated pregnancy one is post-dated but term another is post-dated which is truly post-term.. various variables noted were Age, parity, H/O postdate, onset of labour, mode of delivery, intrapartum complications, fetal weight, Apgar score at 1 and 5 minute, colour of the liquor, admission in NICU, and neonatal death.

All pregnant women with gestational age of $(\geq 40$ week and $\geq 42$ week) admitted for delivery were enrolled in the study. Data was collected on a structured proforma from the hospital admission record and was checked from the emergency room. The cases fulfilling the inclusion criteria were selected for the study. The cases were followed in the ANC ward, $1^{\text {st }}$ stage room, the labour room, O.T., NICU and as well as postnatal ward. They were grouped according to their period of gestation and mode of delivery - Vaginal or instrumental or Cesarean Section. Any Intrapartum complications and maternal and fetal outcome were tabulated. Control group was taken as completed 37 weeks till 40 weeks with comparable parity either primi and multigravida. The control group was taken immediately after each study case matching with parity. Rates and proportion were calculated accordingly with the help of the statistician and the final analysis was done using the appropriate method. These findings were then presented in tables, graphs diagrams. $P$ value was considered significant if $\mathrm{p}<0.05$ SPSS software was used for calculation and tabulation of data. The final results were discussed and the conclusion was derived. The recommendation was then made. Ethical committee approval was taken from Institutional ethical committee.

\section{RESULTS}

\begin{tabular}{cccc} 
Table 1: Incidence of prelabour rupture of membrane (PROM) based on gestational age \\
\cline { 2 - 4 } Total hospital admission & \multicolumn{3}{c}{ Prelabour rupture of membrane } \\
\cline { 2 - 4 } & $28^{+0}-33^{+6}$ wks $n(\%)$ & $34^{+0}-36^{+6}$ wks $n(\%)$ & $37^{+0}-41^{+6}$ wks \\
& $43(\%)$ & $254(7.42)$ \\
\hline
\end{tabular}

Table 2: Incidence of aetiology between gestational age $28^{+0}-36^{+6}$ weeks and $37^{+0}-41^{+6}$ weeks.

\begin{tabular}{|c|c|c|}
\hline \multirow[t]{2}{*}{ Aetiology } & $\begin{array}{l}28^{+0}-36^{+6} \\
{\left[N^{*}=148\right]}\end{array}$ & $\begin{array}{c}37^{+0}-41^{+6} \\
{\left[N^{* *}=254\right]}\end{array}$ \\
\hline & $n(\%)$ & $n(\%)$ \\
\hline Infec & $51(34.45)$ & $24(9.44)$ \\
\hline No infective & $97(65.55)$ & $230(90.56)$ \\
\hline
\end{tabular}

Table 3: Incidence of booked and unbooked case

\begin{tabular}{|c|c|c|}
\hline & $\begin{array}{c}\text { PROM } \\
{\left[N^{*}=402\right]}\end{array}$ & $\begin{array}{c}\text { Control } \\
{\left[N^{* *}=402\right]}\end{array}$ \\
\hline & $n(\%)$ & $n(\%)$ \\
\hline Booked & $62(15.42)$ & $86(21.39)$ \\
\hline Unbooked & $340(84.58)$ & $316(78.61)$ \\
\hline
\end{tabular}


Table 4: Mode of delivery

\begin{tabular}{cc}
\hline Mode of delivery & No $(\%)$ \\
\hline Vaginal vertex & $215(53.5)$ \\
Vaginal assisted Breech delivery & $45(11.2)$ \\
Instrumental & $10(2.5)$ \\
Caesarean section & $132(32.8)$ \\
\hline
\end{tabular}

Table 5. Incidence of chorioamnionitis between gestational age $28^{+0}-36^{+6}$ weeks and $37^{+0}-41^{+6}$ weeks

\begin{tabular}{|c|c|c|c|}
\hline & \multicolumn{2}{|c|}{ Period of gestation } & p Value \\
\hline & $\begin{array}{l}28^{+0}-36^{+6} \\
{\left[N^{*}=148\right]}\end{array}$ & $\begin{array}{c}37^{+0}-41^{+6} \\
{\left[\mathrm{~N}^{* *}=254\right]}\end{array}$ & \\
\hline & $n(\%)$ & $\mathrm{n}(\%)$ & \\
\hline Chorioamnionitis & $37(25)$ & $21(8.26)$ & \\
\hline
\end{tabular}

Table 6: Comparison of Neonatal outcomes in early preterm PROM on Aggressive vs Conservative management

\begin{tabular}{cccc}
\hline Neonatal outcomes & $\begin{array}{c}\text { Aggressive } \\
\mathrm{N}=\mathbf{2 5}\end{array}$ & $\begin{array}{c}\text { Conservative } \\
\mathrm{N}=\mathbf{2 2}\end{array}$ & $*$ p Value \\
\hline Birth weight (mean in $\mathrm{kg})$ & $1.4 \mathrm{~kg}$ & $1.6 \mathrm{Kg}$ & 0.001 \\
Neonatal morbidity $\mathrm{n}(\%)$ & $12(48.0)$ & $5(22.7)$ & 0.01 \\
Neonatal death $\mathrm{n}(\%)$ & $09(36.0)$ & $2(9.09)$ & 0.01 \\
\hline
\end{tabular}

Table 7: Comparison of Neonatal outcomes in late preterm PROM on Aggressive vs Conservative management

\begin{tabular}{cccc}
\hline Neonatal outcomes & $\begin{array}{c}\text { Aggressive } \\
\mathrm{N}=\mathbf{2 3}\end{array}$ & $\begin{array}{c}\text { Conservative } \\
\mathrm{N}=81\end{array}$ & $\mathrm{p}$ Value \\
\hline Birth weight (mean in $\mathrm{kg})$ & $2 \mathrm{~kg}$ & $2.4 \mathrm{~kg}$ & 0.01 \\
Neonatal morbidity $\mathrm{n}(\%)$ & $7(30.4)$ & $6(7.4)$ & 0.01 \\
Neonatal death $\mathrm{n}(\%)$ & $6(26.1)$ & $3(3.7)$ & 0.001 \\
\hline
\end{tabular}

Table 8: Comparison of neonatal morbidities between women with (study group), and without prelabour rupture of membranes (control)

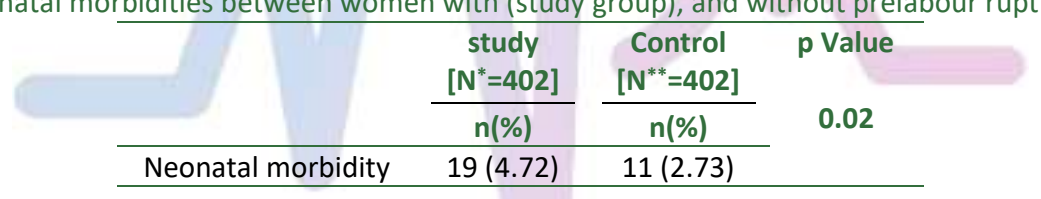

\section{DISCUSSION}

The management of postdated pregnancy is highly debatable. The two managementoptions advocated are expectant management with antepartum fetal surveillance and induction of labour. Again, the timing of induction of labour is difficult to determine and is based on institutional practice. My study was conducted to determine the optimum time of effecting delivery in prolonged pregnancies to improve feto- maternal outcome. Table 1. shows the incidence of term prelabour rupture of membrane was $6.62 \%$, late preterm prelabour rupture of membrane was $2.8 \%$ and early preterm prelabour rupture of membrane was $1.1 \%$. To know the incidence of etiological factors, we divide the study cases in two groups. First group includes preterm PROM (28wks-36wks 6days) and term PROM (37wks-41wks 6days). Aetiological factors are divided into two groups infective and noninfective. For study of infective causes, we took sample from amniotic fluid and from high vaginal swab for cultures. The finding of organism obtained from amniotic fluid culture were corroborated to that of vaginal swab cultures. Study revealed that infective cause is more evident in preterm PROM than term PROM. Table 3. shows, out of 402 cases in the study group, 62 cases were booked and 340 cases were unbooked. In the control group out of 402,86 were booked and 316 were unbooked. From the above table it was also observed that majority of women in the both group were unbooked. The incidence of unbooked cases were higher among cases than control ( $84.58 \%$ vs $78.61 \%)$. Table 4 . shows that the maximum number of PROM women underwent vaginal deliveries (53.5\%). 32.8\% women underwent operative deliveries (forceps and caesarean section). Forceps deliveries were indicated in 10 cases of PROM. It was undertaken for fetal distress in second stage of labour. Other indications were non progress of presenting part and/or maternal distress in second stage of labour. Caesarean section was indicated in chorioamnionitis, fetal distress, malpresentation and failure of induction of labour. Study conductcd in Southern India by Bhat AR et al. found that significant increase in 
rate of instrumental delivery and cesarean delivery was seen beyond 40 weeks of gestation. Rate of cesarean delivery increased from $8.4 \%$ at 39 weeks to $14.9 \%$ at $40^{+6}$ weeks and $20 \%$ at $41^{+6}$ weeks. ${ }^{11}$ Ohel $\mathrm{G}$ et al. from Israel in the retrospective analysis of 2776 pregnancies have found that incidence of emergency cesarean section, mainly due to fetal distress and failure of progression of labour was seen in 11-42 weeks pregnancies. Statistically significant increase in rate of instrumental vaginal delivery, meconium in liquor, and macrosomia was seen in post date group. ${ }^{12}$ The study of liquor amnii shows the higher number of women with features of chorioamnionitis in PROM were below 37weeks of gestational age. The finding of Microorganism obtained from amniotic fluid were corroborated to that of vaginal swab cultures. So, it suggests that chorioamnionitis is due to ascending infection. Table 6 . shows that neonatal morbidity and mortality are lower with conservative compared to aggressive management. Because this was an observational study the morbidity and mortality of aggressively managed, babies was high because of the indication of termination of pregnancy. Table 7 . shows that neonatal morbidity and mortality are lower with conservative compared to aggressive management. Marahatta et al. have found $5.6 \%$ babies born in posttenn period had birth weight more than 4000 grams. Cheng YW et al. in gestation wise analysis have noted that the risk of fetal weight more than 4000 grams increases from 39 weeks onwards. The risk was $7.88 \%$ at 39 weeks which increased to 12.64 at 40 weeks and two and half fold increased (19.23\%) at 41 weeks. Similarly increase in risk of birth weight of more than 4500 grams was 0.92 at 39 weeks and increased to 1.63 at 40 weeks and 3.09 at 41 weeks. ${ }^{13}$ The odds ratio of having macrosomic baby was 1.63 at 40 weeks, which increased to 3.43 at 41 weeks and at 42 weeks it doubled to $7.04^{14}$. Macrosomia and meconium staining was found to be a marker for neonatal complications in the study Caughey AB et al. ${ }^{14}$. Neonatal complications resulted from asphyxia, admission to NICU, septicaemia, conjunctivitis, pneumonia, skin infection. In study cases out of 402 cases 19 babies developed such complication in the neonatal period. Where is in control group only 11 babies developed such complications.

\section{CONCLUSION}

Adverse maternal like caesarean section, instrumental delivery, postpartum haemorrhage and perineal injury was found to higher in postdate group. These were more seen in mother's beyond 42 weeks period of gestation than 40 weeks to $42^{\text {nd }}$ weeks. However, a larger scale study is recommended to study the accurate pregnancy outcome.

\section{REFERENCES}

1. FIGO International classification of Disease. Update, Int'1 J Obstet Gynecol 1980: 17:634.

2. ACOG Practice Bulletin no. 55. Management of Postterm Pregnancy. Obstet Gynecol 2004;104:639-646.

3. Ballantync JW. The problem of the postmature infant. J Obstet Gynecol BrEmp 1902;ii:521-54.

4. Bhat RA. Kushtagi P. A re-look at the duration of human pregnancy. Sign MED j 2006;47(12):1044.

5. Bjerkedal T, Bakketeig LS 1975 Medical Registration of Births in Norway during the 5-year period 1967-71. Time trends and differences between counties and municipalities. Institute of Hygiene and social medicine, University of Bergen, Bergen. Norway. P 71.

6. Maruhatta R, Tuladhar H, Sharma S. Comparative study of post term and term pregnancy in Nepal Medical College Teaching Hospital. Nepal Med Coll J 2009;11(1): 57-60.

7. Ingemarsson I, Kallen. Stillbirths and rate of neonatal deaths in 76,761postterm pregnancies in Sweden. 19821991: a register study. Acta Obstet Gynecol 1997:76 658662.

8. Anner K, Evgenij B, Jon O, Andrej M. Secular trend in pregnancy outcome in 1980-1999 in Komi republic, Russia Int'I J Circumpolar Health 2007: 5:66-68.

9. Gardosi J, Vanner T. Francis A. Gestational age and induction of labour for prolonged pregnancy. Br J Obstet Gynecol. July 1997:10-1: 792-797.

10. Saito M, Yazawa K. Hashiguchi A, et al... Time of ovulation and prolonged pregnancy. Am J Obstet Gynecol 1972;112(1 ): 31-8.

11. Bhat RA. Kushtagi P. A re-look at the duration of human pregnancy Singapore Med J 2006; 47(12): 1044-1048.

12. Ohel G, Yaacobi N, Linder N. et al... Postdate antenatal testing International Journal of Gynaecology Obstetrics; 40 (1005): 145-147.

13. Cheng YW Nicholson JM, Nakagawa S, et al... Perinatal outcomes in low-risk term pregnancies: do they differ by week of gestation ? Am J Obstet Gynecol 2008; 199: 170.el-370.e7.

14. Caughey AB. Musci TJ. Complications of Term Pregnancies Beyond37 Weeks of Gestation. Am J Obstet Gynecol 2004;103 (1):57-62.

Source of Support: None Declared
Conflict of Interest: None Declared

Policy for Articles with Open Access:

Authors who publish with MedPulse International Journal of Gynaecology, (Print ISSN: 2579-0870) (Online ISSN: 2636-4719) agree to the following terms: Authors retain copyright and grant the journal right of first publication with the work simultaneously licensed under a Creative Commons Attribution License that allows others to share the work with an acknowledgement of the work's authorship and initial publication in this journal.

Authors are permitted and encouraged to post links to their work online (e.g., in institutional repositories or on their website) prior to and during the submission process, as it can lead to productive exchanges, as well as earlier and greater citation of published work. 